\title{
Dose-to-medium vs. dose-to-water: Dosimetric evaluation of dose reporting modes in Acuros XB for prostate, lung and breast cancer
}

\author{
Suresh Rana ${ }^{1}$, Shyam Pokharel ${ }^{2}$ \\ ${ }^{I}$ Department of Medical Physics, ProCure Proton Therapy Center, Oklahoma City, Oklahoma, USA \\ ${ }^{2}$ Department of Radiation Oncology, $21^{\text {st }}$ Century Oncology, Naples, Florida, USA
}

Received October 25, 2014; Revised December 05, 2014; Accepted December 10, 2014; Published Online December 13, 2014

\section{Original Article}

\begin{abstract}
Purpose: Acuros XB (AXB) dose calculation algorithm is available for external beam photon dose calculations in Eclipse treatment planning system (TPS). The AXB can report the absorbed dose in two modes: dose-to-water ( $\left.D_{w}\right)$ and dose-to-medium $\left(D_{m}\right)$. The main purpose of this study was to compare the dosimetric results of the AXB_Dm with that of AXB_Dw on real patient treatment plans. Methods: Four groups of patients (prostate cancer, stereotactic body radiation therapy (SBRT) lung cancer, left breast cancer, and right breast cancer) were selected for this study, and each group consisted of 5 cases. The treatment plans of all cases were generated in the Eclipse TPS. For each case, treatment plans were computed using AXB_Dw and AXB_Dm for identical beam arrangements. Dosimetric evaluation was done by comparing various dosimetric parameters in the AXB_ $\mathrm{D}_{\mathrm{w}}$ plans with that of AXB_Dm plans for the corresponding patient case. Results: For the prostate cancer, the mean planning target volume (PTV) dose in the AXB_D plans was higher by up to $1.0 \%$, but the mean PTV dose was within $\pm 0.3 \%$ for the SBRT lung cancer. The analysis of organs at risk (OAR) results in the prostate cancer showed that AXB_D w plans consistently produced higher values for the bladder and femoral heads but not for the rectum. In the case of SBRT lung cancer, a clear trend was seen for the heart mean dose and spinal cord maximum dose, with AXB_D plans producing higher values than the AXB_Dm plans. However, the difference in the lung doses between the AXB_Dm and AXB_Dw plans did not always produce a clear trend, with difference ranged from $-1.4 \%$ to $2.9 \%$. For both the left and right breast cancer, the AXB_Dmplans produced higher maximum dose to the PTV for all cases. The evaluation of the maximum dose to the skin showed higher values in the AXB_Dm plans for all 5 left breast cancer cases, whereas only 2 cases had higher maximum dose to the skin in the AXB_Dm plans for the right breast cancer. Conclusion: The preliminary dosimetric results from our clinical study showed that the selection of either $D_{m}$ or $D_{w}$ in $A X B$ is less likely to produce significant dosimetric differences in the clinical environment. However, the difference between the AXB_Dm and AXB_Dw calculations depends on the disease site, and even for the same type of disease (e.g., lung cancer), the results are patient specific.
\end{abstract}

Keywords: Acuros XB; Dose-to-Medium; Dose-to-Water; Dose Calculation; Heterogeneity Correction

\section{Introduction}

In conventional radiation therapy treatment planning systems (TPS), photon dose calculation algorithms typically report the absorbed dose as dose-to-water $\left(D_{w}\right)$. Dose calculation algorithms employed in the TPS aim to best match the computed results with the measurements, which are performed in water phantoms. In recent years, there has been significant interest in using dose calculation algorithms that are based on Monte Carlo (MC) approach, which can report the absorbed dose in dose-to-medium $\left(\mathrm{Dm}_{\mathrm{m}}\right)$ mode. In the $\mathrm{D}_{\mathrm{m}}$ mode, the absorbed dose is computed to the medium contained in the dose voxel of the material. Siebers et al. ${ }^{1}$ suggested that the conversion of $D_{m}$ to $D_{w}$ may be desirable in some of the situations when MC-based calculations are used in external beam photon radiation therapy. Currently, dosi- metric calibration protocols of external beam photon radiation therapy ${ }^{2,3}$ are based on the $D_{w}$ mode, and the use of either $D_{m}$ or $D_{w}$ (after the conversion of $D_{m}$ to $D_{w}$ ) for MC-based photon dose calculations remains a debating topic. ${ }^{4}$

Varian's Eclipse TPS (Varian Medical Systems, Palo Alto, $\mathrm{CA}$ ) has implemented Acuros XB (AXB) dose calculation algorithm, which can report the absorbed dose in both the $\mathrm{D}_{\mathrm{m}}$ and $\mathrm{D}_{\mathrm{w}}$ options. The AXB utilizes the Linear Boltzmann Transport Equation (LBTE) and solves numerically that describes the macroscopic behavior of ionizing particles as they travel through and interact with matter. ${ }^{5}$ For the AXB_Dm, the macroscopic energy deposition cross-section and atomic

Corresponding author: Suresh Rana; Department of Medical Physics, ProCure Proton Therapy Center, Oklahoma City, Oklahoma, USA. 
density are based on the material properties of local voxel, whereas for the AXB_D $D_{w}$, the energy deposition cross-sections are used for the local media. ${ }^{5-7}$ Detail descriptions on $\mathrm{AXB}$ can be found in the published literature. ${ }^{5-7}$

Several investigators ${ }^{5-31}$ have studied the dosimetric impact of $\mathrm{AXB}$ using $\mathrm{D}_{\mathrm{m}}$ reporting mode, and compared the $A X B \_D_{m}$ results with $\mathrm{MC}$ simulations, measurements, and Anisotropic Analytical Algorithm (AAA) calculations. Few studies have reported the difference between the $D_{m}$ and $D_{w}$ in AXB using MC simulations ${ }^{23}$, measurements ${ }^{24,25,26}$, and computed tomography (CT) data of real patients for nasopharygeal carcinoma ${ }^{26}$ (planning technique used: intensity modulated radiation therapy (IMRT)), and soft-tissue sarcoma $^{27}$ (planning technique used: volumetric modulated arc therapy (VMAT)). Literature review shows that studies comparing AXB_Dm and AXB_Dw calculations on real clinical cases are limited. Hence, it is essential to further investigate if the selection of either $D_{m}$ or $D_{w}$ in AXB will make a significant dosimetric impact in the clinical environment. The major purpose of this study was to compare the dosimetric results of the $A X B \_D_{m}$ calculations with that of AXB_ $D_{w}$ calculations on real patient treatment plans for the prostate, lung, and breast cancer.

\section{Methods and Materials}

\section{Patient selection and CT simulation}

In this retrospective study, all dosimetric data were obtained from $21^{\text {st }}$ Century Oncology, Naples, Florida, USA. The patient selection was done based on the localizations of the tumor, which included prostate cancer (Group 1), stereotactic body radiation therapy (SBRT) lung cancer (Group 2), right breast cancer (Group 3), and left breast cancer (Group 4). Each group consisted five cases previously treated with external beam photon radiation therapy at $21^{\text {st }}$ Century Oncology, Naples, Florida, USA. All patients underwent standard CT simulation on a Phillips Brilliance CT Scanner. The CT scans were acquired using $512 \times 512$ pixels and $2.5 \mathrm{~mm}$ slice thickness. After the CT simulation, DICOM CT images were transferred to the Eclipse TPS for contouring and planning purpose.

\section{Contouring}

All four groups of patients included the planning target volume (PTV), which was expanded from the clinical target volume (CTV) drawn by the physician. Additionally, organs at risk (OARs) were delineated based on the axial CT images. The OARs of each group of patients are provided in Table 1.

\section{Treatment planning}

Treatment plans of all four groups of patients were generated in the Eclipse TPS (version 11.2) using RapidArc, IMRT, and 3D conformal radiation therapy (3DCRT) (Energy: $6 \mathrm{MV}$ X-ray; Machine: TrueBeam (Varian Medical Systems, Palo Alto, CA, USA)). For prostate cases, the total dose prescribed to the PTV was $81 \mathrm{~Gy}$ with a daily dose of $1.8 \mathrm{~Gy}$ in 45 fractions. The prostate cancer treatment plans were generated using a single-full-arc. For the SBRT lung cases, the total dose prescribed to the PTV was 50-60 Gy with a daily dose of 10 or 12 Gy in 5 fractions. The SBRT lung cancer treatment plans were generated using 5-field IMRT $(\mathrm{n}=4)$ and 2-partial-arc RapidArc ( $\mathrm{n}=1)$ techniques.

For the right breast cancer cases, the total dose prescribed to the PTV was $45 \mathrm{~Gy}(\mathrm{n}=4)$ and $46.8 \mathrm{~Gy}(\mathrm{n}=1)$ with a daily dose of $1.8 \mathrm{~Gy}$. The treatment plans were generated using 2-tangent fields IMRT $(\mathrm{n}=4)$ and 2-tangent fields 3DCRT (n $=1$ ) techniques. For all 5 left breast cancer cases, the total dose prescribed to the PTV was 45 Gy with a daily dose of $1.8 \mathrm{~Gy}$ in 25 fractions. The treatment plans were generated using 2-tangent fields IMRT $(\mathrm{n}=3)$ and 2-partial-arc Rapi$\mathrm{dArc}(\mathrm{n}=2)$ techniques. The isocenter in all treatment plans was placed at the center of the PTV.

The treatment plans of all four groups of patients were optimized using the Varian Eclipse Progressive Resolution Optimizer (version 11.2) with an objective of meeting the planning objectives of institutional guidelines. After the optimization process, each treatment plan was calculated using AXB_Dm (version 11.2) and AXB_Dw (version 11.2). The dose calculation grid size was set to $2 \mathrm{~mm}$ for all the cases. The calculated treatment plans were normalized such that the prescribed dose covered at least $95 \%$ of the PTV.

TABLE 1: Organs at risk (OARs) for four different groups of patients.

\begin{tabular}{ccccc}
\hline \hline & Prostate Cancer & SBRT Lung Cancer & Left Breast Cancer & Right Breast Cancer \\
& (Group 1) & (Group 2) & (Group 3) & Contra-Breast \\
\hline \multirow{3}{*}{ OARs } & Rectum & Ipsi-Lung & Contra-Breast & Ipsi-Lung \\
& Bladder & Contra-Lung & Ipsi-Lung & Contra-Lung \\
& Femoral Heads & Heart & Contra-Lung & Heart \\
& & Spinal Cord & Heart & Skin \\
\hline \hline
\end{tabular}

Abbreviations: Ipsi = Ipsi-lateral; Contra $=$ Contra-lateral; SBRT $=$ Stereotactic Body Radiation Therapy 


\section{Plan evaluation}

The dose-volume histograms (DVHs) of both the AXB_Dm and $A X B \_D_{w}$ were generated in the Eclipse TPS. Treatment plans were evaluated for various dosimetric parameters. For a comparative purpose, the relative difference $(\Delta)$ in the corresponding dosimetric parameter (for example, mean dose, maximum dose, etc.) between the AXB_Dm and AXB_Dw plans of the same case was calculated using Equation 1.

$\Delta(x)=\frac{A X B_{-} D_{w}(x)-A X B_{-} D_{m}(x)}{A X B_{-} D_{w}(x)} \times 100$-------(1)

where, $x$ is the corresponding dosimetric parameter in the $A X B \_D_{w}$ and $A X B \_D m$ plans of the same case.

\section{Results}

The dosimetric results in the AXB_Dm and AXB_Dw plans of all four groups of patients are presented in Figures 1-4.

\section{Prostate cancer}

For the prostate cancer, in comparison to the AXB_Dm plans, the $A X B \_D_{w}$ plans produced a higher mean dose to the PTV $\left(\Delta_{\text {avg }}=0.8 \%\right)$, rectum $\left(\Delta_{\text {avg }}=0.4 \%\right)$, bladder $\left(\Delta_{\text {avg }}=1.4 \%\right)$, and femoral heads $\left(\Delta_{\text {avg }}=2.5 \%\right)$. The relative rectal volume exposed to radiation was higher in the AXB_Dw plans with the average relative differences of $0.6 \%$ for $\mathrm{V}_{30}, 0.9 \%$ for $\mathrm{V}_{50}$, and $1.3 \%$ for $V_{70}$. Similarly, the relative bladder volume exposed to radiation was higher in the AXB_Dw plans with the average relative differences of $1.4 \%$ for $\mathrm{V}_{30}, 2.3 \%$ for $\mathrm{V}_{50}$, and $4.5 \%$ for $\mathrm{V}_{70}$.

\section{SBRT lung cancer}

For SBRT lung cancer, the mean dose in the AXB_Dw plans was consistently higher for the heart $\left(\Delta_{\text {avg }}=1.3 \%\right)$ and esophagus $\left(\Delta_{\text {avg }}=0.7 \%\right)$ but almost identical for the PTV $\left(\Delta_{\text {avg }}=-0.1 \%\right)$. In evaluating the mean dose to the ipsi-lung and contra-lung, no clear trend was observed, with AXB_Dw plans producing higher values in 2 cases for the ipsi-lung and in 3 cases for the contra-lung. For the ipsi-lung, the $V_{5}, V_{10}$, and $V_{20}$ were similar in the AXB_Dw and AXB_Dm, with an average relative difference within $\pm 0.4 \%$. For the $V_{5}$ of the contra-lung, the averaged difference is $-0.2 \%$ (range, $-1.3 \%$ to $0.1 \%$ ) with higher values in the AXB_Dw plans in 3 cases. The maximum dose to the spinal cord was consistently higher $\left(\Delta_{\text {avg }}=2.1 \%\right)$ in the AXB_Dw plans, with a relative difference ranged from $1.4 \%$ to $4.4 \%$.

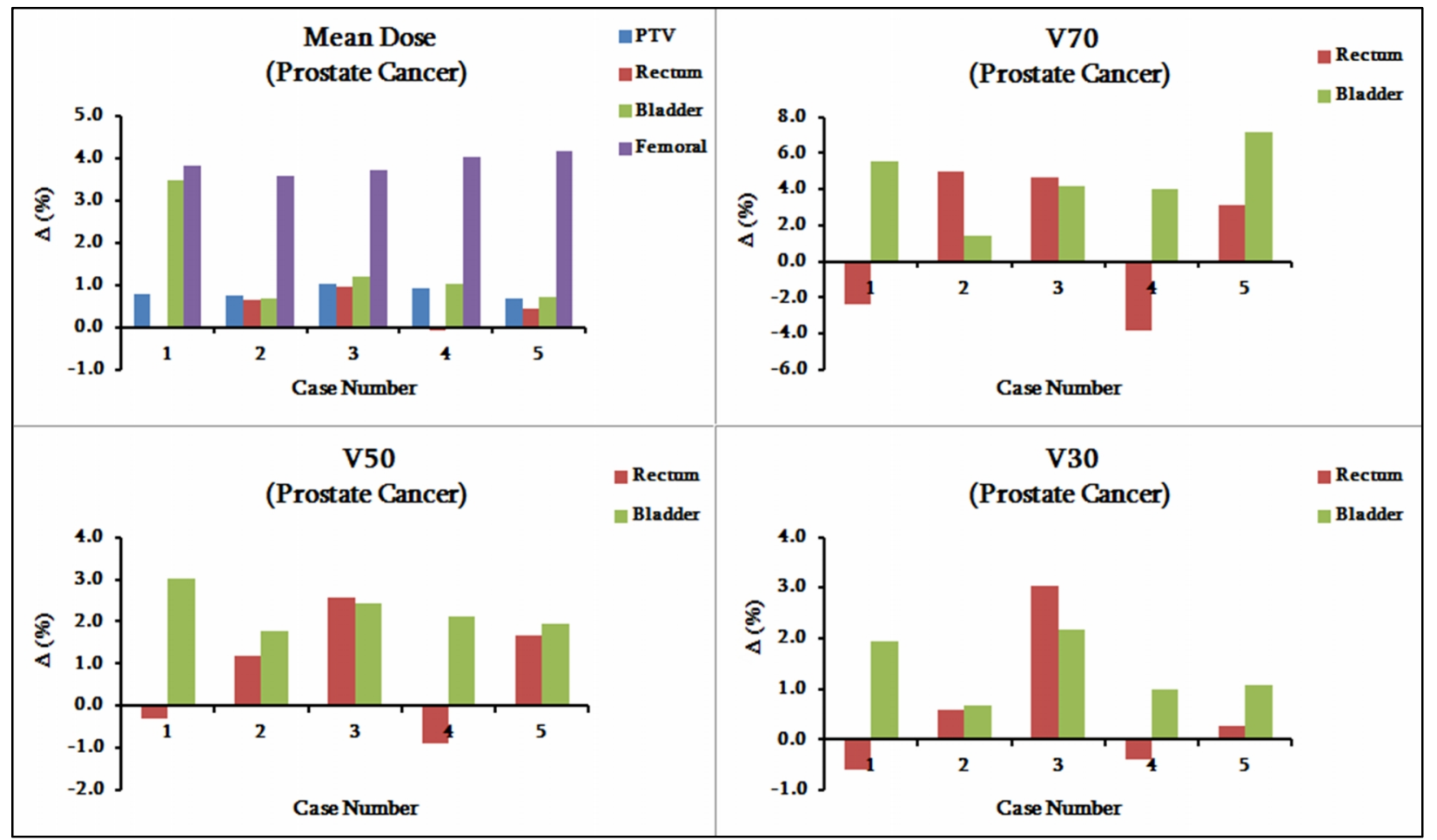

FIG. 1: Relative difference $(\Delta)$ in dosimetric results between the $A X B_{-} D_{\mathrm{w}}$ and $A X B_{-} D_{m}$ plans for 5 different prostate cancer cases. $V_{\mathrm{n}}$ means percentage volume irradiated by $\mathrm{n}$ Gy or more of a certain structure. The $\Delta$ is defined in Equation 1 . 


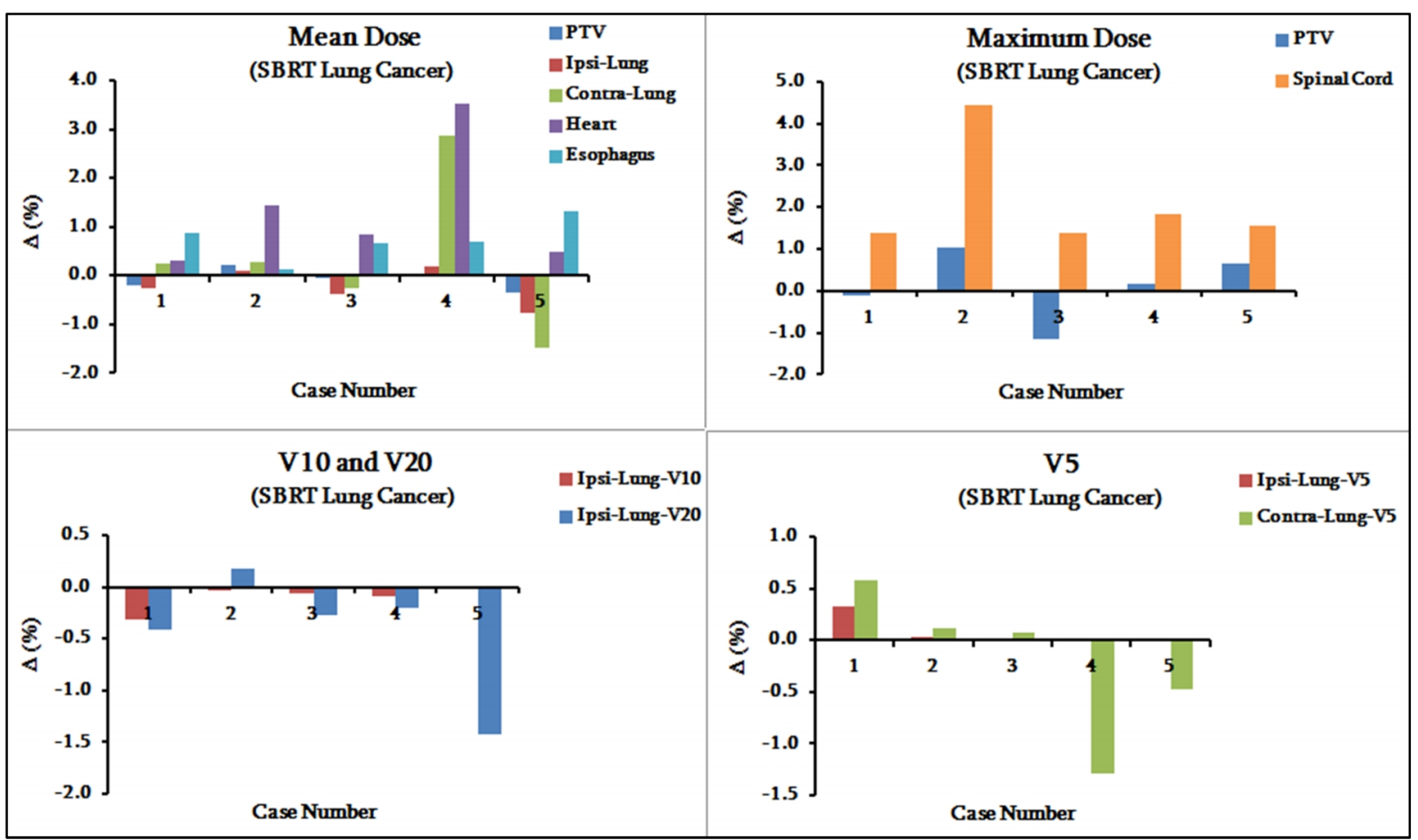

FIG. 2: Relative difference $(\Delta)$ in dosimetric results between the AXB_D $D_{w}$ and AXB_Dm plans for 5 different SBRT lung cases. $V_{n}$ means percentage volume irradiated by $\mathrm{n}$ Gy or more of a certain structure. The $\Delta$ is defined in Equation 1.

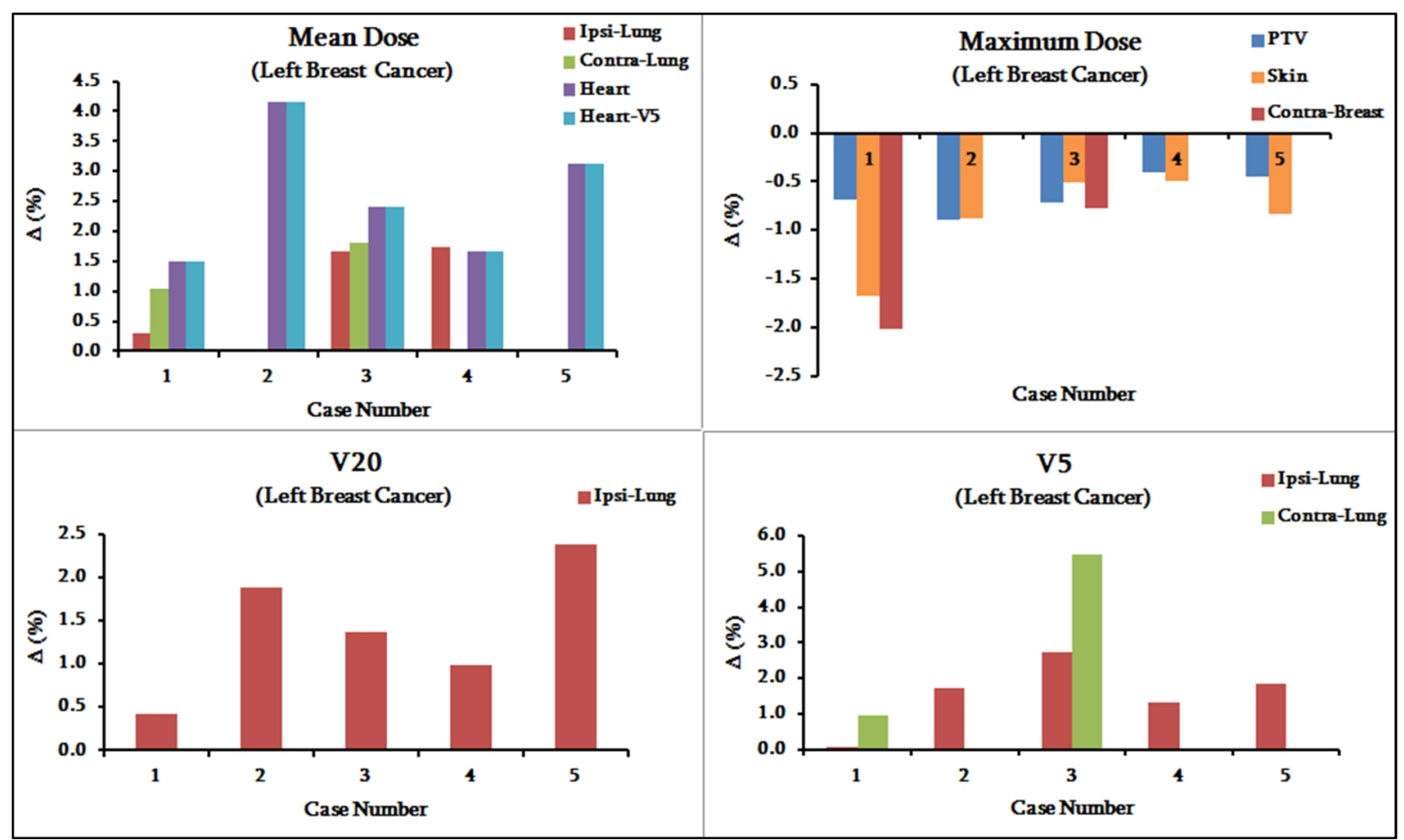

FIG. 3: Relative difference $(\Delta)$ in dosimetric results between the AXB_D $\mathrm{w}_{\mathrm{w}}$ and AXB_Dm plans for 5 different left breast cancer cases. $V_{\mathrm{n}}$ means percentage volume irradiated by $\mathrm{n}$ Gy or more of a certain structure. The $\Delta$ is defined in Equation 1. 


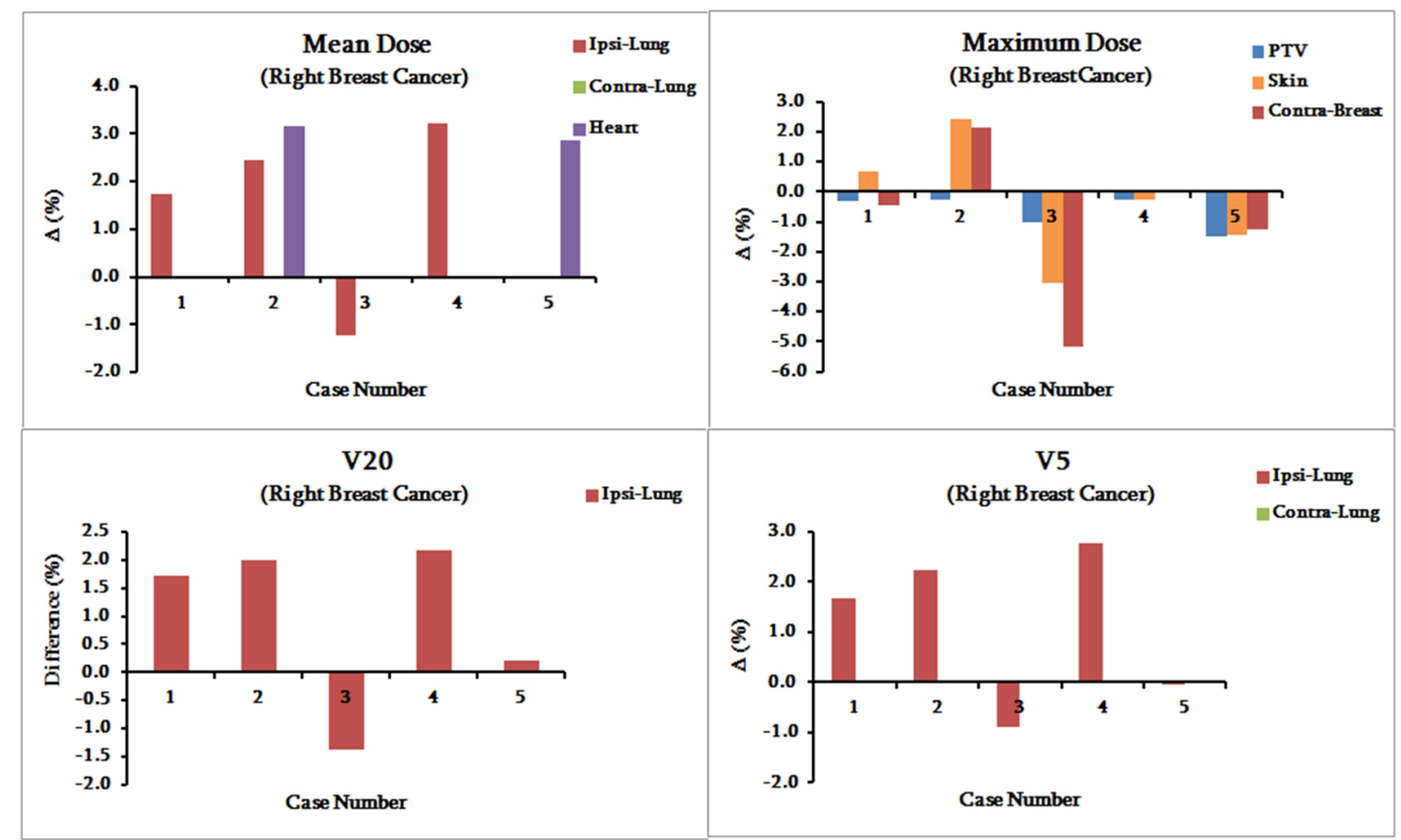

FIG. 4: Relative difference $(\Delta)$ in dosimetric results between the AXB_D w and AXB_Dm plans for 5 different right breast cancer cases. $V_{n}$ means percentage volume irradiated by $\mathrm{n}$ Gy or more of a certain structure. The $\Delta$ is defined in Equation 1.

\section{Left breast cancer}

For the left breast cancer, the difference in the PTV maximum dose between the AXB_Dw and AXB_Dm plans ranged from $-0.4 \%$ to $-0.9 \%$. On average, the mean dose in the AXB_Dw plans was higher for the ipsi-lung $\left(\Delta_{\text {avg }}=0.7 \%\right)$, contra-lung $\left(\Delta_{\text {avg }}=0.6 \%\right)$, and heart $\left(\Delta_{\text {avg }}=2.6 \%\right)$, whereas AXB_Dm plans had higher values for the maximum dose to the skin $\left(\Delta_{\text {avg }}=-0.9 \%\right)$ and contra-breast $\left(\Delta_{\text {avg }}=-0.6 \%\right)$. For the ipsi-lung, the AXB_Dw plans had higher values in the case of $\mathrm{V}_{5}\left(\Delta_{\text {avg }}=1.5 \%\right)$ and $\mathrm{V}_{20}\left(\Delta_{\text {avg }}=1.2 \%\right)$.

\section{Right breast cancer}

For the right breast cancer, the difference in the PTV maximum dose between the AXB_Dw and AXB_Dm plans ranged from $-0.3 \%$ to $-1.5 \%$. The mean dose in the $A X B \_D_{w}$ plans was higher for the ipsi-lung $\left(\Delta_{\text {avg }}=1.2 \%\right)$ and almost identical for the majority of the cases for the contra-lung and heart (except for case \#2 and \#5). On average, the AXB_Dm plans had higher values for the maximum dose to the skin $\left(\Delta_{\text {avg }}=-0.3 \%\right)$ and contra-breast $\left(\Delta_{\text {avg }}=-0.9 \%\right)$. In the case of ipsi-lung, on average, the AXB_Dw plans had higher values for the $\mathrm{V}_{5}\left(\Delta_{\mathrm{avg}}=1.2 \%\right)$ and $\mathrm{V}_{20}\left(\Delta_{\mathrm{avg}}=0.8 \%\right)$ when compared to the AXB_Dm plans.

\section{Discussion}

The dosimetric impact of $\mathrm{D}_{\mathrm{m}}$ and $\mathrm{D}_{\mathrm{w}}$ reporting mode in $\mathrm{AXB}$ was investigated for the prostate, SBRT lung, and breast cancer. The AXB_Dm and AXB_Dw plans were evaluated based on the results derived from the DVH in the Eclipse TPS. The preliminary results from the clinical cases in this study showed that the differences in dosimetric results between the AXB_Dm and AXB_Dw plans depend on the tumor type. For instance, the mean PTV dose in the AXB_D plans was slightly higher (relative difference up to $1.0 \%$ ) for the prostate cancer when compared to the mean PTV dose in the AXB_Dw plans for the SBRT lung cancer (relative difference within $\pm 0.3 \%$ ). Kan et al. ${ }^{26}$ and Fogliata et al. ${ }^{27}$ also reported higher values calculated by AXB_Dw when compared to the ones calculated by AXB_Dm.

The analysis of the OAR results for the prostate cancer (Figure 1) showed that the AXB_Dw plans consistently produced higher values for the bladder and femoral heads but not for the rectum. In the case of SBRT lung (Figure 2), a clear trend was seen for the heart mean dose and spinal cord maximum dose, with $A X B \_D_{w}$ plans producing higher values when compared to the $A X B \_D_{m}$ plans. However, the difference in the lung doses between the AXB_Dm and AXB_Dw plans did not always produce a clear trend, with the relative difference ranged from $-1.4 \%$ to $2.9 \%$. For both the left and right breast cancer, we observed higher maximum dose to the PTV as 
well as higher mean dose to the heart in the AXB_Dmplans, and this was true for all cases. The evaluation of the maximum dose to the skin showed higher values in the AXB_Dm plans for all 5 left breast cancer cases, whereas only 2 cases had higher maximum dose to the skin in the AXB_Dm plans for the right breast cancer cases.

The results presented in this study demonstrated that the difference between the AXB_Dm and AXB_Dw calculations is dependent on the tumor type, and even for the same type of tumor, the results are patient specific. The use of $D_{m}$ vs. $D_{w}$ for external beam photon radiation therapy is an interesting debating topic for the medical physics community. ${ }^{4}$ On one hand, the supporters of $D_{m}$ reason that (i) conversion from $\mathrm{D}_{\mathrm{m}}$ to $\mathrm{D}_{\mathrm{w}}$ adds uncertainty in dose calculations due to uncertainties in computed stopping power ratios; (ii) changing to $\mathrm{D}_{\mathrm{m}}$ will have minimal impact on the treatment protocols; (iii) $\mathrm{D}_{\mathrm{m}}$ is more likely to provide a better measure of biological response; and (iv) conversion of the $D_{m}$ to $D_{w}$ defeats a potential advantage of using MC-based dose calculation algorithms. On the other hand, the supporters of $D_{w}$ argue that (i) commissioning beam data are always measured in water; (ii) clinical experience in terms of tumor/tissue response is based on $\mathrm{D}_{\mathrm{w}}$; (iii) dosimetry calibration protocols are based in water; and (iv) conversion from CT numbers to media results uncertainty in the medium type and composition.

\section{Conclusion}

The preliminary dosimetric results from our clinical study showed that the selection of either $D_{m}$ or $D_{w}$ in AXB is less likely to produce significant dosimetric differences in the clinical environment. However, the difference between the AXB_Dm and AXB_Dw calculations depends on the disease site, and even for the same type of disease (e.g., lung cancer), the results are patient specific. Future studies need to include large cohort of clinical cases with different types of cancer. Also, it is recommended to investigate the dosimetric impact of the treatment technique (e.g., 3DCRT, IMRT, and VMAT) on the AXB_Dm and AXB_Dw calculations for different types of cancer.

\section{Conflict of interest}

The authors declare that they have no conflicts of interest. The authors alone are responsible for the content and writing of the paper.

\section{References}

1. Siebers JV, Keall PJ, Nahum AE, Mohan R. Converting absorbed dose to medium to absorbed dose to water for Monte Carlo based photon beam dose calculations. Phys Med Biol 2000; 45:983-95.
2. Almond PR, et al. AAPM's TG-51 protocol for clinical reference dosimetry of high-energy photon and electron beams. Med Phys 1999; 26 1847-70.

3. Huq MS, Andreo P, Song H. Comparison of the IAEA TRS-398 and AAPM TG-51 absorbed dose to water protocols in the dosimetry of high-energy photon and electron beams. Phys Med Biol 2001; 46 2985-3006.

4. Keall P, Liu H. Dm rather than Dw should be used in Monte Carlo treatment planning. Med Phys 2002; 29: 922-4.

5. Vassiliev O, Wareing T, McGhee J, et al. Validation of a new grid based Blotzmann equation solver for dose calculation in radiotherapy with photon beams. Phys Med Biol 2010; 55:581-98.

6. Han T, Mikell JK, Salehpour M, Mourtada F. Dosimetric comparison of Acuros XB deterministic radiation transport method with Monte Carlo and model-based convolution methods in heterogeneous media. Med Phys 2011; 38:2651-64.

7. Bush K, Gagne IM, Zavgorodni S, et al. Dosimetric validation of Acuros XB with Monte Carlo methods for photon dose calculations. Med Phys 2011; 38:2208-21.

8. Kroon PS, Hol S, Essers M. Dosimetric accuracy and clinical quality of Acuros XB and AAA dose calculation algorithm for stereotactic and conventional lung volumetric modulated arc therapy plans. Radiat Oncol 2013; 8:149.

9. Kathirvel M, Subramanian S, Clivio A, et al. Critical appraisal of the accuracy of Acuros-XB and Anisotropic Analytical Algorithm compared to measurement and calculations with the compass system in the delivery of RapidArc clinical plans. Radiat Oncol 2013; 8:140.

10. Rana S, Rogers K. Dosimetric evaluation of Acuros $\mathrm{XB}$ dose calculation algorithm with measurements in predicting doses beyond different air gap thickness for smaller and larger field sizes. JMed Phys 2013; 38:9-14.

11. Rana S, Rogers K, Lee T, et al. Verification and Dosimetric Impact of Acuros XB Algorithm for Stereotactic Body Radiation Therapy (SBRT) and RapidArc Planning for Non-Small-Cell Lung Cancer (NSCLC) Patients. Int Jour of Med Phys Clin Eng Rad Onc 2013; 2:6-14.

12. Stathakis S, Esquivel C, Quino L, et al. Accuracy of the Small Field Dosimetry Using the Acuros XB Dose Calculation Algorithm within and beyond Heterogeneous Media for $6 \mathrm{MV}$ Photon Beams. Int Jour of Med Phys Clin Eng Rad Onc 2012; 1:78-87.

13. Rana S, Rogers K, Pokharel S, Cheng C. Evaluation of Acuros XB algorithm based on RTOG 0813 dosimetric criteria for SBRT lung treatment with RapidArc. J Appl Clin Med Phys 2014; 15:4474. 
14. Lloyd SA, Ansbacher W. Evaluation of an analytic linear Boltzmann transport equation solver for high-density inhomogeneities. Med Phys 2013; 40:011707.

15. Kan MW, Leung LH, Yu PK. Dosimetric impact of using the Acuros XB algorithm for intensity modulated radiation therapy and RapidArc planning in nasopharyngeal carcinomas. Int J Radiat Oncol Biol Phys 2013; 85:e73-80.

16. Liu HW, Nugent Z, Clayton R, et al. Clinical impact of using the deterministic patient dose calculation algorithm Acuros XB for lung stereotactic body radiation therapy. Acta Oncol 2014; 53:324-9.

17. Mißlbeck M, Kneschaurek P. Comparison between Acuros XB and Brainlab Monte Carlo algorithms for photon dose calculation. Strahlenther Onkol 2012; 188:599-605.

18. Fogliata A, Nicolini G, Clivio A, et al. Critical appraisal of Acuros XB and Anisotropic Analytic Algorithm dose calculation in advanced non-small-cell lung cancer treatments. Int J Radiat Oncol Biol Phys 2012; 83:1587-95.

19. Hoffmann L, Jørgensen MB, Muren LP, Petersen JB. Clinical validation of the Acuros XB photon dose calculation algorithm, a grid-based Boltzmann equation solver. Acta Oncol 2012; 51:376-85.

20. Fogliata A, Nicolini G, Clivio A, et al. Accuracy of Acuros XB and AAA dose calculation for small fields with reference to RapidArc stereotactic treatments. Med Phys 2011; 38: 6228-37.

21. Fogliata A, Nicolini G, Clivio A, et al. On the dosimetric impact of inhomogeneity management in the Acuros XB algorithm for breast treatment. $R a-$ diat Oncol 2011; 6:103.

22. Fogliata A, Nicolini G, Clivio A, et al. Dosimetric validation of the Acuros XB Advanced Dose Calculation algorithm: fundamental characterization in water. Phys Med Biol 2011; 56:1879-904.
23. Fogliata A, Nicolini G, Clivio A, et al. Dosimetric evaluation of Acuros XB Advanced Dose Calculation algorithm in heterogeneous media. Radiat Oncol 2011; 6:82.

24. Han T, Followill D, Mikell J, et al. Dosimetric impact of Acuros XB deterministic radiation transport algorithm for heterogeneous dose calculation in lung cancer. Med Phys 2013; 40:051710.

25. Han T, Mourtada F, Kisling K, et al. Experimental validation of deterministic Acuros XB algorithm for IMRT and VMAT dose calculations with the Radiological Physics Center's head and neck phantom. Med Phys 2012; 39:2193-202.

26. Kan MW, Leung LH, So RW, Yu PK. Experimental verification of the Acuros XB and AAA dose calculation adjacent to heterogeneous media for IMRT and RapidArc of nasopharygeal carcinoma. Med Phys 2013; 40:031714.

27. Fogliata A, Scorsetti M, Navarria P, et al. Dosimetric comparison between VMAT with different dose calculation algorithms and protons for soft-tissue sarcoma radiotherapy. Acta Oncol 2013; 52:545-52.

28. Ojala JJ, Kapanen MK, Hyödynmaa SJ, et al. Performance of dose calculation algorithms from three generations in lung SBRT: comparison with full Monte Carlo-based dose distributions. J Appl Clin Med Phys 2014; 15:4662.

29. Ojala J, Kapanen M, Sipilä P, et al. The accuracy of Acuros XB algorithm for radiation beams traversing a metallic hip implant - comparison with measurements and Monte Carlo calculations. J Appl Clin Med Phys 2014; 15:4912.

30. Rana S. Clinical dosimetric impact of Acuros XB and analytical anisotropic algorithm (AAA) on real lung cancer treatment plans: review. Int J Cancer Ther Oncol 2014; 2:02019.

31. Ojala J. The accuracy of the Acuros XB algorithm in external beam radiotherapy - a comprehensive review. Int J Cancer Ther Oncol 2014; 2:020417. 